\title{
Effect of catheter-based renal denervation on left ventricular function, mass and (un)twist with two-dimensional speckle tracking echocardiography
}

\author{
Lida Feyz $^{1}$ - Bas M. van Dalen ${ }^{1}$ Marcel L. Geleijnse ${ }^{1}$ Nicolas M. Van Mieghem ${ }^{1}$. \\ Ron T. van Domburg ${ }^{1} \cdot$ Joost Daemen $^{1}$
}

Received: 20 July 2016/Revised: 13 April 2017/Accepted: 24 April 2017/Published online: 11 May 2017

(C) The Author(s) 2017. This article is an open access publication

\begin{abstract}
Background Speckle tracking echocardiography (STE) is an echocardiography modality that is able to measure left ventricular (LV) characteristics, including rotation, strain and strain rate. Strain measures myocardial fibre contraction and relaxation. This study aims to assess the effect of renal sympathetic denervation (RDN) on functional myocardial parameters, including STE, and to identify potential differences between responders and nonresponders.

Methods The study population consisted of 31 consecutive patients undergoing RDN in the context of treatment for resistant hypertension. Patients were included between December 2012 and June 2014. Transthoracic echocardiography and speckle tracking analysis was performed at baseline and at 6 months follow-up.

Results The study population consisted of 31 patients with treatment-resistant hypertension treated with RDN (mean age $64 \pm 10$ years, 15 men). The total study population could be divided into responders $(n=19)$ and non-responders $(n=12)$ following RDN. RDN reduced office blood pressure by $18.9 \pm 26.8 / 8.5 \pm 13.5 \mathrm{mmHg}$ $(p<0.001)$. A significant decrease was seen in LV posterior wall thickness (LVPWd) $\quad(0.47 \pm 1.0 \mathrm{~mm}$; $p=0.020$ ), without a significant change in the LV mass index (LVMI). In the total cohort, only peak late diastolic filling velocity (A-wave velocity) decreased significantly by $5.3 \pm 13.2 \mathrm{~cm} / \mathrm{s} \quad(p=0.044)$ and peak untwisting
\end{abstract}

Joost Daemen

j.daemen@erasmusmc.nl

1 Department of Cardiology, Thoraxcenter, Erasmus University Medical Center, P.O. Box 2040, 3000 CA Rotterdam, The Netherlands velocity decreased significantly by $14.5 \pm 28.9 \% \mathrm{~s}$ $(p=0.025)$.

Conclusion RDN reduced blood pressure and significantly improved functional myocardial parameters such as A-wave velocity and peak untwisting velocity in patients with treatment-resistant hypertension, suggesting a potential beneficial effect of RDN on myocardial mechanics.

Keywords Speckle tracking echocardiography - Twist . Strain $\cdot$ Renal sympathetic denervation

\section{Background}

Hypertension is associated with a significantly increased risk for adverse cardiac and cerebrovascular events, as well as chronic kidney disease [1]. Despite optimal medical treatment, blood pressure control often remains poor and the risk for cardiovascular disease remains high. With prevalence ranging between 15 and 30\%, treatment-resistant hypertension remains an important medical challenge and leads to intrinsic changes in the heart muscle and is associated with left ventricular (LV) hypertrophy and diastolic dysfunction [2, 3].

Catheter-based renal denervation (RDN) has been introduced as a treatment modality to optimise blood pressure control in patients with treatment-resistant hypertension by reducing sympathetic nerve activity. Unfortunately, the exact blood pressure-lowering effect of renal sympathetic denervation remains disputed, demonstrated by non-responder rates varying between 8 and $37 \%$, depending on study-specific cohorts and definitions used [4]. However, sympathetic hyperactivity has been directly associated with LV remodelling and heart rate, which makes it imperative to look at the effects of renal 
sympathetic denervation beyond blood pressure [5]. Speckle tracking echocardiography (STE) is a new echocardiography modality that is able to measure LV characteristics, including rotation, strain and strain rate. Strain measures myocardial fibre contraction and relaxation [6]. This study aims to assess the effect of RDN on functional myocardial parameters, including STE, and to identify potential differences between responders and nonresponders.

\section{Methods}

\section{Study population}

The study population consisted of 31 consecutive patients undergoing RDN in the context of treatment for resistant hypertension according to recent recommendations [7]. Patients were included between December 2012 and June 2014. All patients underwent non-invasive pre-procedural renal artery imaging and were discussed in a multi-disciplinary team including interventional cardiologists, radiologists and hypertension specialists. As part of routine practice, all patients referred for RDN underwent extensive blood and urine analyses, 24-h ambulatory blood pressure measurement (24 h ABPM), echocardiography, echocardiogram (ECG) and magnetic resonance imaging (MRI) to assess renal artery eligibility and exclude renal artery stenosis in order to be able to exclude secondary causes of hypertension. Informed consent was obtained from all patients.

\section{Definitions and end-points}

Office blood pressure measurements were recorded three times in a resting situation with intervals of 5 min using an Omron automated blood pressure monitor. Patients were classified as responders in case the drop in 6 months office systolic blood pressure was $10 \mathrm{mmHg}$ or higher. In order to identify subtle changes in LV function, STE was used to obtain apical rotation, basal rotation, LV twist, twist velocity, peak untwisting velocity, time to peak untwisting velocity, global longitudinal strain (GLS), global circumferential strain (GCS), peak early and late longitudinal diastolic strain rate, and peak early and late circumferential diastolic strain rate. LV twist is defined as the maximal value of the apical systolic rotation-basal systolic rotation [8].

\section{Transthoracic echocardiography}

Echocardiography measurements were performed before the RDN procedure (baseline) and 6 months after the RDN procedure. Two-dimensional grey-scale images were obtained in the left lateral decubitus position using a commercially available ultrasound system (iE33, Philips, Best, The Netherlands), equipped with a broadband (1-5 MHz) S5-1 transducer (frequency transmitted 1.7 MHz, received 3.4 MHz). Data were analysed by two experienced echocardiographers according to the recent recommendations [9]. The following echocardiographic parameters were acquired: LV end-diastolic septal (LVSd) and posterior wall thickness (LVPWd), and LV end-diastolic (LVEDD) and end-systolic dimension (LVESD). LV mass was calculated with the Devereux formula [10]. Body surface area (BSA) was calculated according to the Mosteller formula [11]. LV mass was indexed by BSA as recommended in the guidelines [12].

From the mitral inflow pattern, peak early (E-wave velocity) and late (A-wave velocity) filling velocities, E/A ratio and E-wave velocity deceleration time were measured. Tissue Doppler was applied end-expiratory in the pulsed-wave Doppler mode at the level of the inferoseptal side of the mitral annulus from an apical four-chamber view, to obtain Em septal (peak early diastolic wave velocity of the mitral annulus) and $\mathrm{E} / \mathrm{Em}$ ratio.

To acquire the highest wall tissue velocities, the angle between the Doppler beam and the longitudinal motion of the investigated structure was adjusted to a minimal level. The spectral pulsed-wave Doppler velocity range was adjusted to obtain appropriate scale.

To optimise STE, the settings were adjusted to obtain a frame rate of 50-70 frames/s. The echo images were transformed to a QLAB Advanced Quantification Software workstation (version 10.0, Philips, Best, The Netherlands) for offline analysis.

\section{Speckle tracking analysis}

STE is an approved echocardiographic modality that provides information on regional and global ventricular function [13]. In order to obtain this information, apical long-axis views (four-, three- and two-chamber views) and parasternal short-axis views (at the apical, mid-ventricular and basal LV levels) were assessed. The aortic valve closure was assessed in a parasternal long-axis view and added manually. After selecting the appropriate view, the endocardial border was automatically recognised and the tracking points were positioned. When this auto-trace function was not optimal, the tracking points were repositioned manually on an end-diastolic frame. Next, the software automatically tracked these points using speckle tracking. LV ejection fraction was assessed using this automated endocardial border detection. Most components of LV systolic function [rotation (clockwise rotation as viewed from the apical level has a positive value and 
counterclockwise rotation from the basal level has a negative value), twist, global circumferential strain (GCS) and GCS rate (GCSR)] and diastolic function (peak untwisting velocity, time to peak untwisting velocity, peak early and late circumferential diastolic strain rate) were abstracted from parasternal short-axis views, whereas others were derived from the apical views [systolic function: global longitudinal strain (GLS) and GLS rate (GLSR); diastolic function: peak early and late longitudinal diastolic strain rate]. Data were exported to a spreadsheet program (Excel, Microsoft Corporation, Redmond, WA) to determine these parameters. In a previous study, we have demonstrated the reproducibility and variability of the parameters investigated in the current study in our centre [14].

\section{RDN procedure}

Procedures were performed using four different systems: Paradise $^{\mathrm{TM}}$ (ReCor Medical, Palo Alto, CA) $(n=13)$, OneShot $^{\mathrm{TM}}$ (Covidien, Campbell, CA) $(n=3)$, Vessix $\mathrm{V} 2^{\mathrm{TM}}$ (Boston Scientific, Natick, MA) $(n=5)$ and Symplicity $^{\mathrm{TM}}$ (Medtronic, Minneapolis, MN) $(n=10)$. Procedures were performed according to the device-specific instructions for use [7, 15]. All procedures were performed under conscious sedation with midazolam and fentanyl.

\section{Statistical analysis}

All continuous variables are expressed as mean \pm standard deviation (SD). Continuous variables were compared using Student's $t$ test. Simple linear regression of peak untwisting velocity against heart rate was performed. Categorical variables were compared with the Chi square test or Fisher's exact test when appropriate. A $p$-value $<0.05$ was considered statistically significant. The statistical analysis was performed with SPSS software (version 21.0).

\section{Results}

\section{Study population}

Thirty-one patients with resistant hypertension following RDN were enrolled in this study, all of whom completed the 6 months follow-up period. The mean age was $64 \pm 10$ years and 15 patients $(48 \%)$ were male. A total of 19 patients were classified as responders versus 12 nonresponders. Besides a significantly lower age of the responders $(61 \pm 10$ vs. $69 \pm 9$ years in the non-responders; $p=0.028$ ), no significant differences in patient characteristics were seen between both groups (Table 1).

\section{Blood pressure}

A significant decrease was seen in office-based systolic blood pressure (SBP) and diastolic blood pressure (DBP) after RDN at 6 months follow-up: $182 \pm 18$ vs. $163 \pm$ $27 \mathrm{mmHg}(p<0.001)$ and $94 \pm 16$ vs. $85 \pm 14 \mathrm{mmHg}$ ( $p=0.001)$, respectively. The same applied for the systolic $24 \mathrm{~h}$ ABPM after RDN $(150 \pm 12$ vs. $142 \pm$ $18 \mathrm{mmHg} ; p=0.017)$ and the diastolic $24 \mathrm{~h}$ ABPM after $\operatorname{RDN}(83 \pm 13$ vs. $78 \pm 11 \mathrm{mmHg} ; p=0.006)($ Table 2$)$.

\section{Conventional echocardiography and STE}

In the total cohort, significant differences at baseline versus the 6-month follow-up period were noted in LVPWd, A-wave velocity and peak untwisting velocity. LVPWd decreased significantly from $8.8 \pm 1.4 \mathrm{~mm}$ at baseline to $8.3 \pm 1.5 \mathrm{~mm}$ at follow-up $(p=0.020)$. LV mass index (MI) reduced by $3.2 \pm 11.6 \mathrm{~g} / \mathrm{m}^{2}$ at 6 months. A-wave velocity decreased significantly from $69.7 \pm 14.2 \mathrm{~cm} / \mathrm{s}$ at baseline to $64.4 \pm 14.0 \mathrm{~cm} / \mathrm{s}$ at follow-up ( $p=0.044)$. No significant changes were seen in the other conventional echocardiographic parameters. Furthermore, peak untwisting velocity decreased significantly from $-70 \pm 28.5 \% \mathrm{~cm}$ at baseline to $-56 \pm 24.9 \% \mathrm{~cm}$ at follow-up $(p=0.025)$ (Table 2).

Stratifying the cohort in responders and non-responders did reveal significant changes from baseline to follow-up in A-wave velocity and peak untwisting velocity in the responders (Fig. 1). In the non-responders, LVESD increased significantly from $37.4 \pm 9.3$ to $39.7 \pm 10.1 \mathrm{~mm}$ ( $p=0.013$ ), although it should be noted that $\mathrm{LV}$ ejection fraction (EF) showed no difference. Also, no differences were found in the more sensitive systolic parameters GLS, GCS and LV twist.

Comparing the baseline characteristics of both cohorts, no difference was observed in the LVMI at baseline in the responders as compared to the non-responders (98 \pm 25.4 vs. $116 \pm 42.9 \mathrm{~g} / \mathrm{m}^{2}$ ) (Table 3$)$.

\section{Predictors for response}

No association was found between the clinical characteristics, conventional echocardiographic and speckle tracking parameters which could predict response.

\section{Discussion}

The aim of this study was to assess if RDN resulted in functional and structural cardiac changes as assessed using both conventional 2D echocardiography and 2D STE. A secondary objective was to assess any differences in these 
Table 1 Clinical characteristics of the study population, responder vs. non-responder at baseline

\begin{tabular}{|c|c|c|c|c|}
\hline & $\begin{array}{l}\text { All patients } \\
\text { Baseline }(n=31)\end{array}$ & $\begin{array}{l}\text { Responder } \\
\text { Baseline }(n=19)\end{array}$ & $\begin{array}{l}\text { Non-responder } \\
\text { Baseline }(n=12)\end{array}$ & $\begin{array}{l}p \text {-Value } \\
\text { Responder vs. } \\
\text { non-responder }\end{array}$ \\
\hline Age (years) & $64 \pm 10$ & $61 \pm 10$ & $69 \pm 9$ & 0.028 \\
\hline Male gender, $n(\%)$ & $15(48)$ & $10(53)$ & $5(42)$ & 0.552 \\
\hline $\operatorname{BMI}\left(\mathrm{kg} / \mathrm{m}^{2}\right)$ & $29 \pm 4$ & $29 \pm 5$ & $28 \pm 3$ & 0.639 \\
\hline Mean office SBP (mmHg) & $182 \pm 18$ & $186 \pm 20$ & $176 \pm 15$ & 0.133 \\
\hline Mean office DBP (mmHg) & $94 \pm 16$ & $97 \pm 14$ & $89 \pm 18$ & 0.149 \\
\hline Mean systolic ABPM (mmHg) & $150 \pm 12$ & $148 \pm 12$ & $152 \pm 13$ & 0.358 \\
\hline Mean diastolic ABPM (mmHg) & $83 \pm 13$ & $83 \pm 11$ & $82 \pm 17$ & 0.946 \\
\hline Heart rate (beats/min) & $68 \pm 12$ & $69 \pm 12$ & $65 \pm 12$ & 0.282 \\
\hline CAD $(\%)$ & $16(52)$ & $12(63)$ & $4(33)$ & 0.106 \\
\hline Atrial fibrillation (\%) & $2(7)$ & $2(11)$ & - & 0.368 \\
\hline \multicolumn{5}{|l|}{ Cardiovascular risk factors, $n(\%)$} \\
\hline Hypercholesterolaemia & $22(71)$ & $15(79)$ & $7(58)$ & 0.204 \\
\hline Current smoker & $11(36)$ & $6(32)$ & $5(42)$ & 0.619 \\
\hline Diabetes mellitus & $8(26)$ & $7(37)$ & $1(8)$ & 0.086 \\
\hline Number of hypertensive drugs & $4 \pm 1$ & $4 \pm 1$ & $4 \pm 1$ & 0.634 \\
\hline \multicolumn{5}{|l|}{ Patients receiving (drug class) $(\%)$} \\
\hline ACE inhibitors/ARBs & $27(87)$ & $17(90)$ & $10(83)$ & 0.507 \\
\hline Direct renin inhibitors & $1(3)$ & - & $1(8)$ & 0.387 \\
\hline Beta-blockers & $25(81)$ & $16(84)$ & $9(75)$ & 0.435 \\
\hline Alpha-blockers & $9(29)$ & $4(21)$ & $5(42)$ & 0.168 \\
\hline Calcium channel blockers & $25(81)$ & $16(84)$ & $9(75)$ & 0.435 \\
\hline Aldosterone antagonist & $4(13)$ & $3(16)$ & $1(8)$ & 0.493 \\
\hline Diuretics & $22(71)$ & $13(68)$ & $9(75)$ & 0.417 \\
\hline Central acting agent & $3(10)$ & $1(5)$ & $2(17)$ & 0.328 \\
\hline
\end{tabular}

Values are mean \pm standard deviation $(\mathrm{SD})$ or $n(\%)$

$B M I$ Body mass index; $S B P$ systolic blood pressure; $D B P$ diastolic blood pressure; $A B P M$ ambulatory blood pressure monitoring; $C A D$ coronary artery disease; $A C E$ angiotensin-converting enzyme; $A R B s$ angiotensin receptor blocker

parameters between responders and non-responders. We observed a significant difference in blood pressure and heart rate at 6 months post procedure. Echocardiographically, at 6 months, a significant difference was noted in LVPWd and A-wave velocity. Additionally, STE demonstrated a significant difference in peak untwisting velocity.

Persistent sympathetic nervous system hyperactivity plays a critical role in hypertension and is associated with significant structural and functional cardiac changes [16]. In this study, we found that RDN significantly reduced office blood pressure by $18.9 \pm 26.8 / 8.5 \pm 13.5 \mathrm{mmHg}$ $(p<0.001)$ and heart rate by $4.5 \pm 9.9$ beats $/ \mathrm{min}$ $(p=0.016)$. In line with these findings, we observed a significant decrease in LVPWd $(0.47 \pm 1.0 \mathrm{~mm})$, along with a reduction in LVMI of $3.2 \pm 11.6 \mathrm{~g} / \mathrm{m}^{2}$ in the total cohort. The fact that this reduction did not reach statistical significance could be due to a lack of power.
Looking further into diastolic function, which is strongly related to hypertension and blood pressure control, revealed a pseudonormal diastolic dysfunction, based on normal E/A ratio, but increased left atrial (LA) dimension and $\mathrm{E} / \mathrm{Em}$ ratio in the overall study population. We observed a significant decrease in the A-wave velocity by $5.3 \pm 13.2 \mathrm{~cm} / \mathrm{s}(p=0.044)$ in the total population. In the responders, the difference in the A-wave velocity even decreased to $8.5 \pm 13.8 \mathrm{~cm} / \mathrm{s}(p=0.027)$. The decrease in A-wave velocity after renal denervation could implicate an improvement in the LV relaxation and a subsequent better diastolic function [17]. Additionally, a pseudonormal diastolic function may reflect a decrease in LV compliance and a moderate increase in LA pressure in our population, with impaired relaxation and prolonged A-wave velocity before treatment. The link between peak untwisting velocity and A-wave velocity may be explained by the rate of uncoiling. In other words, it is likely that less force is 
Table 2 Clinical, echocardiographic and speckle tracking parameters in patients following renal denervation

\begin{tabular}{|c|c|c|c|}
\hline & Baseline & Follow-up (6 months) & $p$-Value \\
\hline \multicolumn{4}{|l|}{ Clinical parameters } \\
\hline Mean office SBP (mmHg) & $182 \pm 18$ & $163 \pm 27$ & $<0.001$ \\
\hline Mean office DBP (mmHg) & $94 \pm 16$ & $85 \pm 14$ & 0.001 \\
\hline Mean systolic ABPM (mmHg) & $150 \pm 12$ & $142 \pm 18$ & 0.017 \\
\hline Mean diastolic ABPM (mmHg) & $83 \pm 13$ & $78 \pm 11$ & 0.006 \\
\hline Heart rate (beats/min) & $68 \pm 12$ & $63 \pm 11$ & 0.016 \\
\hline \multicolumn{4}{|l|}{ Echocardiographic parameters } \\
\hline LA size $(\mathrm{mm})$ & $45.1 \pm 7.9$ & $44.5 \pm 6.8$ & 0.523 \\
\hline LAVI $\left(\mathrm{mL} / \mathrm{m}^{2}\right)$ & $36.8 \pm 10.9$ & $35.6 \pm 11.0$ & 0.335 \\
\hline IVSd (mm) & $10.8 \pm 2.1$ & $10.3 \pm 1.8$ & 0.105 \\
\hline LVPWd (mm) & $8.8 \pm 1.4$ & $8.3 \pm 1.5$ & 0.020 \\
\hline LVEDD (mm) & $53.8 \pm 8.1$ & $55.5 \pm 7.9$ & 0.028 \\
\hline LVESD (mm) & $37.7 \pm 8.7$ & $39.5 \pm 8.3$ & 0.048 \\
\hline $\operatorname{LVEF}(\%)$ & $59.5 \pm 11.0$ & $58.1 \pm 10.1$ & 0.123 \\
\hline LVMI $\left(\mathrm{g} / \mathrm{m}^{2}\right)$ & $105.8 \pm 33.8$ & $102.6 \pm 30.2$ & 0.133 \\
\hline \multicolumn{4}{|l|}{ Doppler indices } \\
\hline $\mathrm{E}(\mathrm{cm} / \mathrm{s})$ & $67.2 \pm 20.5$ & $65.0 \pm 23.1$ & 0.519 \\
\hline $\mathrm{A}(\mathrm{cm} / \mathrm{s})$ & $69.7 \pm 14.2$ & $64.4 \pm 14.0$ & 0.044 \\
\hline $\mathrm{E} / \mathrm{A}$ ratio & $1.0 \pm 0.3$ & $1.0 \pm 0.4$ & 0.557 \\
\hline DET (ms) & $219.7 \pm 51.6$ & $219.7 \pm 84.3$ & 0.996 \\
\hline Em septal $(\mathrm{cm} / \mathrm{s})$ & $5.5 \pm 1.8$ & $5.1 \pm 1.7$ & 0.134 \\
\hline $\mathrm{E} / \mathrm{Em}$ ratio & $12.3 \pm 4.7$ & $12.5 \pm 3.0$ & 0.840 \\
\hline \multicolumn{4}{|l|}{ Speckle tracking echocardiography } \\
\hline $\operatorname{GLS}(\%)$ & $-19.6 \pm 4.2$ & $-19.9 \pm 3.5$ & 0.653 \\
\hline GCS $(\%)$ & $-27.3 \pm 6.5$ & $-27.2 \pm 5.3$ & 0.877 \\
\hline Early GLSR & $0.89 \pm 0.25$ & $0.89 \pm 0.21$ & 0.932 \\
\hline Late GLSR & $0.80 \pm 0.23$ & $0.82 \pm 0.24$ & 0.495 \\
\hline Early GCSR & $1.88 \pm 0.62$ & $1.80 \pm 0.51$ & 0.470 \\
\hline Late GCSR & $1.39 \pm 0.50$ & $1.32 \pm 0.44$ & 0.432 \\
\hline Apical GR $\left(^{\circ}\right)$ & $4.8 \pm 3.4$ & $4.3 \pm 2.7$ & 0.542 \\
\hline Basal GR $\left(^{\circ}\right)$ & $-4.2 \pm 2.0$ & $-3.5 \pm 2.2$ & 0.212 \\
\hline Twist $\left({ }^{\circ}\right)$ & $8.7 \pm 4.4$ & $7.4 \pm 3.7$ & 0.178 \\
\hline Twist velocity ( $\%$ s) & $15.5 \pm 12.9$ & $13.7 \pm 12.1$ & 0.665 \\
\hline Peak untwisting velocity $(\% / s)$ & $-70.6 \pm 28.5$ & $-56.1 \pm 24.9$ & 0.025 \\
\hline Time to peak untwisting velocity (s) & $0.08 \pm 0.07$ & $0.09 \pm 0.08$ & 0.707 \\
\hline LVEDV (mL) & $76.1 \pm 28.4$ & $82.6 \pm 34.2$ & 0.152 \\
\hline LVESV (mL) & $30.5 \pm 17.9$ & $33.9 \pm 22.9$ & 0.146 \\
\hline LVEF $(\%)$ & $61.8 \pm 10.1$ & $61.5 \pm 8.5$ & 0.803 \\
\hline
\end{tabular}

Values are mean $\pm \mathrm{SD}$

$S B P$ Systolic blood pressure; $D B P$ diastolic blood pressure; $A B P M$ ambulatory blood pressure monitoring; $L A$ left atrial; $L A V I$ left atrial volume indexed; IVSd interventricular septum thickness (diastole); $L V P W d$ left ventricular posterior wall thickness (diastole); $L V E D D$ left ventricular end-diastolic dimension; $L V E S D$ left ventricular end-systolic dimension; $L V E F$ left ventricular ejection fraction; $L V M I$ left ventricular mass index; $E$ peak early phase filling velocity; $A$ peak atrial phase filling velocity; $D E T$ deceleration time; $E m$ peak early wave velocity; $G L S$ global longitudinal strain; $G C S$ global circumferential strain; $G L S R$ global longitudinal strain rate (early and late diastole); GCSR global circumferential strain rate (early and late diastole); GR global rotation (apical and basal level). Twist is defined as the instantaneous left ventricular peak systolic twist. The peak untwisting velocity is the peak diastolic de-rotation velocity. $E D V$ Enddiastolic volume; $E S V$ end-systolic volume 
Fig. 1 Change in A-wave velocity and peak untwisting velocity at baseline and 6 months follow-up, categorised by responders and nonresponders

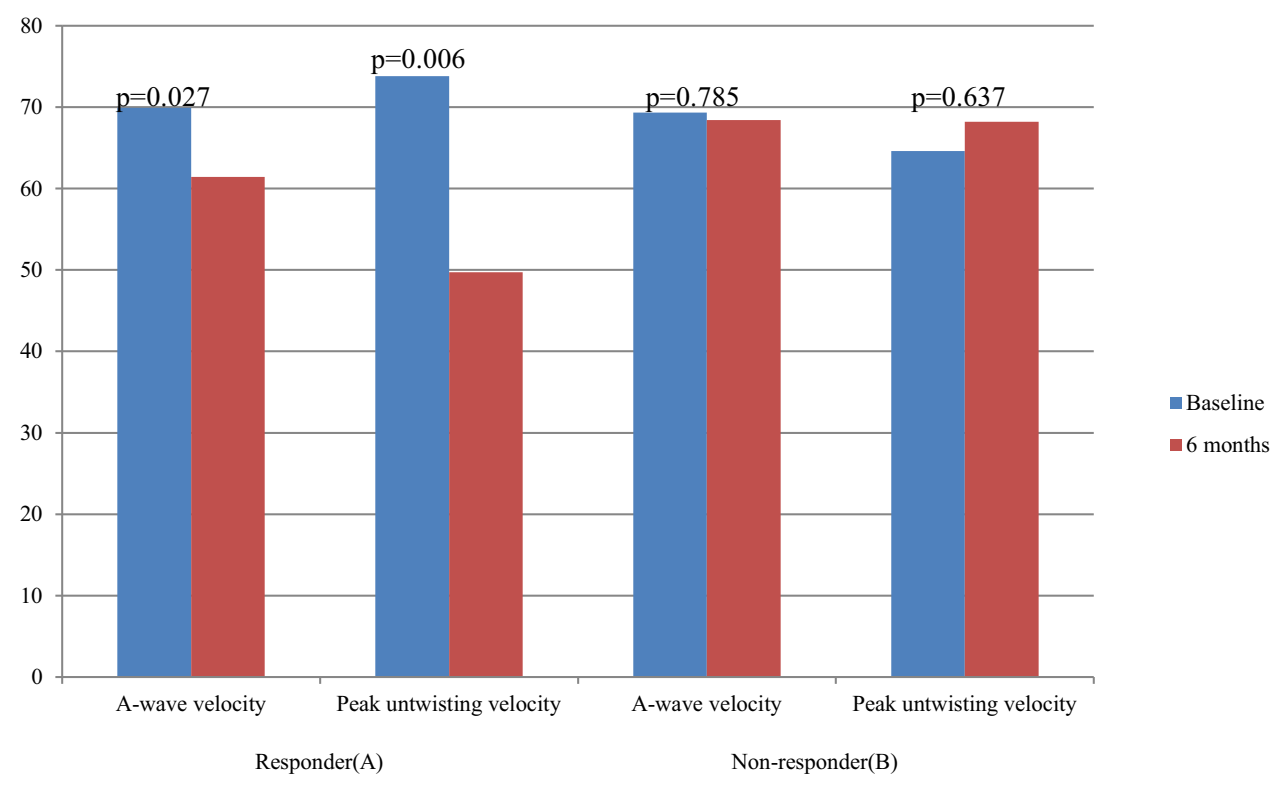

needed for the active atrial contraction during late diastolic filling after blood pressure lowering. The relation between LV untwisting and the conventional parameters was also described in previous work, in which a positive correlation between untwisting rate and A-wave velocity was found, while there was no correlation between E-wave velocity and untwisting rate [8]. However, these findings should be interpreted with caution, as no differences were noted in other echocardiographic parameters determining diastolic function, such as the E/Em ratio and LA dimensions.

Hypertensive patients have a greater risk of developing cardiac fibrosis, myocyte hypertrophy and diastolic dysfunction [18]. These changes may influence the LV twist and rotation [19]. LV twist is a wringing motion of the heart as the apex rotates with respect to the base around the LV long axis, which is a key element for regulating LV systolic and diastolic mechanics [20]. LV twist is derived from the dynamic interaction between subendocardial and subepicardial myocardial fibres, the latter defining the direction of the LV twist. It is known that, in myocardial fibrosis and LV hypertrophy, which is related to pressure overload, impairment appears frequently in the subendocardial layer, leading to a dominance of the subepicardial fibres [21]. This might explain the increased LV twist in patients with LV hypertrophy. LV untwisting starts after the peak LV twist. In a healthy population, the peak systolic twist is supposed to store potential energy and is thought to contribute towards diastolic suction and facilitate early LV diastolic filling. Previous work from our group demonstrated that peak untwisting velocity is increased in hypertrophic cardiomyopathy patients with mild diastolic dysfunction, as well as in aortic stenosis patients [8, 22]. Our study is the first to demonstrate a significant decrease in peak untwisting velocity of $14.5 \pm 28.9 \%(p=0.025)$ in patients undergoing RDN. This change was mainly driven by a change in peak untwisting velocity by $24.1 \pm 28.7^{\circ} / \mathrm{s}(p=0.006)$ in the responders. In nonresponders, no difference following RDN was observed. The decrease in untwisting velocity after renal denervation could implicate an improvement in the LV relaxation and a subsequent better diastolic function, similar to A-wave velocity. One may hypothesise that, in hypertension patients, like in hypertrophic cardiomyopathy and aortic stenosis patients, increased peak untwisting velocity serves as a compensatory mechanism for abnormal relaxation and prevents the need to increase LA pressure [22, 23]. RDN probably leads to an improvement of these specific changes in $\mathrm{LV}$ rotational and de-rotational mechanics, especially in responders. However, no significant change was seen in twist and twist velocity. Finally, we observed a significant decrease in heart rate following $\mathrm{RDN}$ in our population. This is in line with several randomised controlled studies which also demonstrated a decrease in heart rate following RDN [24, 25]. Interestingly, a similar decrease in heart rate was observed in the (sham) control arm of both studies, suggesting that RDN by itself has no significant effect on heart rate. Additional exploratory analyses in our study ruled out a correlation between heart rate and untwisting velocity $\left(R^{2}=0.1 \%\right)$.

Future studies, comparing hypertensive patients with a healthy control group, may be warranted in order to investigate myocardial geometry changes in hypertensive subjects in the context of diastolic LV twisting and untwisting, blood pressure or heart rate independently. 
Table 3 Baseline and 6-month follow-up parameters in patients following renal denervation, responders vs. non-responders

\begin{tabular}{|c|c|c|c|c|c|c|c|}
\hline & \multicolumn{3}{|c|}{ Responder $(n=19)$} & \multicolumn{3}{|c|}{ Non-responder $(n=12)$} & \multirow{2}{*}{$\begin{array}{l}p \text { value } \\
\text { Responder vs } \\
\text { non-responde } \\
\text { at baseline }\end{array}$} \\
\hline & Baseline & 6 months & $p$ value & Baseline & 6 months & $p$ value & \\
\hline \multicolumn{8}{|l|}{ Echocardiographic parameters } \\
\hline LA size $(\mathrm{mm})$ & $44.6 \pm 8.0$ & $44.2 \pm 7.5$ & 0.712 & $45.8 \pm 7.9$ & $44.9 \pm 5.7$ & 0.585 & 0.706 \\
\hline LAVI $\left(\mathrm{mL} / \mathrm{m}^{2}\right)$ & $37.3 \pm 11.1$ & $36.0 \pm 12.2$ & 0.394 & $36.1 \pm 11.3$ & $34.9 \pm 9.7$ & 0.637 & 0.855 \\
\hline IVSd (mm) & $10.5 \pm 2.0$ & $10.3 \pm 2.2$ & 0.408 & $11.3 \pm 2.2$ & $10.3 \pm 1.2$ & 0.176 & 0.352 \\
\hline LVPWd (mm) & $8.7 \pm 1.6$ & $8.3 \pm 1.8$ & 0.130 & $9.0 \pm 1.2$ & $8.4 \pm 1.2$ & 0.089 & 0.546 \\
\hline LVEDD (mm) & $52.9 \pm 7.8$ & $55.1 \pm 6.8$ & 0.060 & $55.1 \pm 8.7$ & $56.2 \pm 9.7$ & 0.289 & 0.484 \\
\hline LVESD (mm) & $37.9 \pm 8.5$ & $39.4 \pm 7.3$ & 0.284 & $37.4 \pm 9.3$ & $39.7 \pm 10.1$ & 0.013 & 0.872 \\
\hline LVEF (\%) & $57.6 \pm 10.1$ & $57.0 \pm 9.5$ & 0.587 & $62.5 \pm 12.2$ & $59.8 \pm 11.1$ & 0.088 & 0.235 \\
\hline LVMI $\left(\mathrm{g} / \mathrm{m}^{2}\right)$ & $98.8 \pm 25.4$ & $98.8 \pm 25.4$ & a & $116 \pm 42.9$ & $108.5 \pm 36.9$ & 0.136 & 0.151 \\
\hline \multicolumn{8}{|l|}{ Doppler indices } \\
\hline $\mathrm{E}(\mathrm{cm} / \mathrm{s})$ & $72.2 \pm 19.5$ & $70.9 \pm 24.7$ & 0.759 & $59.7 \pm 20.5$ & $56.2 \pm 17.7$ & 0.555 & 0.103 \\
\hline $\mathrm{A}(\mathrm{cm} / \mathrm{s})$ & $69.9 \pm 17.0$ & $61.4 \pm 14.4$ & 0.027 & $69.3 \pm 10.0$ & $68.4 \pm 13.0$ & 0.785 & 0.914 \\
\hline E/A ratio & $1.0 \pm 0.3$ & $1.1 \pm 0.3$ & 0.374 & $0.9 \pm 0.3$ & $0.9 \pm 0.4$ & 0.889 & 0.249 \\
\hline DET (ms) & $207.5 \pm 40.6$ & $189.7 \pm 41.7$ & 0.155 & $238.1 \pm 62.2$ & $264.6 \pm 111.3$ & 0.400 & 0.113 \\
\hline Em septal $(\mathrm{cm} / \mathrm{s})$ & $5.6 \pm 1.6$ & $5.3 \pm 1.7$ & 0.515 & $5.5 \pm 2.1$ & $4.8 \pm 1.8$ & 0.151 & 0.882 \\
\hline E/Em ratio & $12.5 \pm 3.6$ & $12.5 \pm 3.3$ & 0.872 & $12.2 \pm 5.8$ & $12.3 \pm 2.9$ & 0.898 & 0.875 \\
\hline \multicolumn{8}{|l|}{ Speckle tracking echocardiography } \\
\hline Twist $\left(^{\circ}\right)$ & $8.0 \pm 3.8$ & $6.8 \pm 3.8$ & 0.242 & $10.1 \pm 5.2$ & $8.6 \pm 3.5$ & 0.492 & 0.278 \\
\hline Twist velocity ( $\%$ s) & $14.7 \pm 13.2$ & $13.3 \pm 11.2$ & 0.792 & $17.1 \pm 13.2$ & $14.5 \pm 14.5$ & 0.740 & 0.673 \\
\hline Peak untwisting velocity $(\% / s)$ & $-73.8 \pm 30.4$ & $-49.7 \pm 23.8$ & 0.006 & $-64.6 \pm 25.5$ & $-68.2 \pm 23.5$ & 0.637 & 0.475 \\
\hline Time to peak untwisting velocity (s) & $0.07 \pm 0.08$ & $0.09 \pm 0.08$ & 0.431 & $0.10 \pm 0.06$ & $0.08 \pm 0.07$ & 0.509 & 0.389 \\
\hline GLS (\%) & $-19.2 \pm 4.7$ & $-19.5 \pm 3.9$ & 0.661 & $-20.6 \pm 3.0$ & $-20.6 \pm 2.4$ & 0.938 & 0.480 \\
\hline GCS $(\%)$ & $-27.2 \pm 6.7$ & $-26.5 \pm 5.9$ & 0.476 & $-27.5 \pm 6.5$ & $-28.5 \pm 4.2$ & 0.657 & 0.916 \\
\hline Early GLSR & $0.90 \pm 0.28$ & $0.89 \pm 0.19$ & 0.809 & $0.87 \pm 0.21$ & $0.91 \pm 0.26$ & 0.460 & 0.625 \\
\hline Late GLSR & $0.76 \pm 0.25$ & $0.77 \pm 0.24$ & 0.840 & $0.86 \pm 0.19$ & $0.92 \pm 0.22$ & 0.429 & 0.188 \\
\hline Early GCSR & $1.91 \pm 0.56$ & $1.81 \pm 0.50$ & 0.339 & $1.81 \pm 0.75$ & $1.77 \pm 0.57$ & 0.883 & 0.736 \\
\hline Late GCSR & $1.26 \pm 0.53$ & $1.15 \pm 0.33$ & 0.320 & $1.62 \pm 0.34$ & $1.62 \pm 0.48$ & 0.990 & 0.094 \\
\hline
\end{tabular}

Values are mean $\pm \mathrm{SD}$

$L A$ Left atrial; $L A V I$ left atrial volume index; IVSd interventricular septum thickness (diastole); $L V P W d$ left ventricular posterior wall thickness (diastole); $L V E D D$ left ventricular end-diastolic dimension; $L V E S D$ left ventricular end-systolic dimension; $L V E F$ left ventricular ejection fraction; $L V M I$ left ventricular mass index; $E$ peak early phase filling velocity; $A$ peak atrial phase filling velocity; $D E T$ deceleration time; $E m$ peak early wave velocity. Twist is defined as the instantaneous left ventricular peak systolic twist. The peak untwisting velocity is the peak diastolic de-rotation velocity. GLS Global longitudinal strain; GCS global circumferential strain; GLSR global longitudinal strain rate (early and late diastole); GCSR global circumferential strain rate (early and late diastole)

${ }^{\text {a }}$ Standard error of the difference

\section{Limitations}

Data are derived from a small patient population and a lack of power might have impacted our findings. Furthermore, accurate assessment of changes in cardiac systolic and diastolic function and volumes with conventional echocardiography produces limited image quality in patients with a body mass index (BMI) above $25 \mathrm{~kg} / \mathrm{m}^{2}$. In some patients $(n=6)$, automatically detected LV contours had be corrected manually, which might have impacted the reliability of the measurements. Four different renal denervation systems were used. Based on individual previous studies, the blood pressurelowering effect of these individual devices remains in the same range; however, a differential effect on the echocardiographic parameters measured in our study could not be excluded.

\section{Conclusion}

Renal sympathetic denervation (RDN) reduced blood pressure and heart rate, and significantly improved functional myocardial parameters such as A-wave velocity and 
peak untwisting velocity in patients with treatment-resistant hypertension, suggesting potential pleiotropic beneficial effects of renal sympathetic denervation on myocardial mechanics. Further dedicated studies are needed to elucidate the potential role of $\mathrm{RDN}$ on echocardiographic parameters.

\section{Compliance with ethical standards}

Conflict of interest Lida Feyz, Bas M. van Dalen, Marcel L. Geleijnse and Ron T. van Domburg declare that they have no conflict of interest.

Funding Joost Daemen and Nicolas M. Van Mieghem received institutional research support from ReCor Medical. Joost Daemen received institutional research support from Boston Scientific, St. Jude Medical and Medtronic.

Human rights statement All procedures followed were in accordance with the ethical standards of the responsible committee on human experimentation (institutional and national) and with the Helsinki Declaration of 1964 and later revisions.

Informed consent Informed consent was obtained from all patients for being included in the study.

Open Access This article is distributed under the terms of the Creative Commons Attribution 4.0 International License (http://crea tivecommons.org/licenses/by/4.0/), which permits unrestricted use, distribution, and reproduction in any medium, provided you give appropriate credit to the original author(s) and the source, provide a link to the Creative Commons license, and indicate if changes were made.

\section{References}

1. Kearney PM, Whelton M, Reynolds K, Muntner P, Whelton PK, He J. Global burden of hypertension: analysis of worldwide data. Lancet. 2005;365(9455):217-23.

2. Pimenta E, Calhoun DA. Resistant hypertension: incidence, prevalence, and prognosis. Circulation. 2012;125(13):1594-6.

3. Aeschbacher BC, Hutter D, Fuhrer J, Weidmann P, Delacrétaz E, Allemann Y. Diastolic dysfunction precedes myocardial hypertrophy in the development of hypertension. Am J Hypertens. 2001;14(2):106-13.

4. Ukena C, Cremers B, Ewen S, Böhm M, Mahfoud F. Response and non-response to renal denervation: who is the ideal candidate? EuroIntervention. 2013;9(Suppl R):R54-7.

5. Daemen J, Mahfoud F. Renal denervation: expanding the indication. EuroIntervention. 2013;9(Suppl R):R101-4.

6. Blessberger H, Binder T. NON-invasive imaging: two dimensional speckle tracking echocardiography: basic principles. Heart. 2010;96(9):716-22.

7. Tsioufis C, Mahfoud F, Mancia G, Redon J, Damascelli B, Zeller $\mathrm{T}$, et al. What the interventionalist should know about renal denervation in hypertensive patients: a position paper by the ESH WG on the interventional treatment of hypertension. EuroIntervention. 2014;9(9):1027-35.

8. van Dalen BM, Kauer F, Michels M, Soliman OI, Vletter WB, van der Zwaan HB, et al. Delayed left ventricular untwisting in hypertrophic cardiomyopathy. J Am Soc Echocardiogr. 2009;22(12):1320-6.
9. Lang RM, Badano LP, Mor-Avi V, Afilalo J, Armstrong A, Ernande L, et al. Recommendations for cardiac chamber quantification by echocardiography in adults: an update from the American Society of Echocardiography and the European Association of Cardiovascular Imaging. Eur Heart $\mathbf{J}$ Cardiovasc Imaging. 2015;16(3):233-70.

10. Lang RM, Bierig M, Devereux RB, Flachskampf FA, Foster E, Pellikka PA, et al. Recommendations for chamber quantification: a report from the American Society of Echocardiography's Guidelines and Standards Committee and the Chamber Quantification Writing Group, developed in conjunction with the European Association of Echocardiography, a branch of the European Society of Cardiology. J Am Soc Echocardiogr. 2005;18(12):1440-63.

11. Mosteller RD. Simplified calculation of body-surface area. N Engl J Med. 1987;317(17):1098.

12. Lang RM, Badano LP, Mor-Avi V, Afilalo J, Armstrong A, Ernande L, et al. Recommendations for cardiac chamber quantification by echocardiography in adults: an update from the American Society of Echocardiography and the European Association of Cardiovascular Imaging. J Am Soc Echocardiogr. 2015;28(1):1-39.

13. Helle-Valle T, Crosby J, Edvardsen T, Lyseggen E, Amundsen $\mathrm{BH}$, Smith $\mathrm{HJ}$, et al. New noninvasive method for assessment of left ventricular rotation: speckle tracking echocardiography. Circulation. 2005;112(20):3149-56.

14. van Dalen BM, Soliman OI, Vletter WB, Kauer F, van der Zwaan $\mathrm{HB}$, ten Cate FJ, et al. Feasibility and reproducibility of left ventricular rotation parameters measured by speckle tracking echocardiography. Eur J Echocardiogr. 2009;10(5):669-76.

15. Daemen J. Current technologies: an introduction. EuroIntervention. 2013;9(Suppl R):R75-82.

16. Levick SP, Murray DB, Janicki JS, Brower GL. Sympathetic nervous system modulation of inflammation and remodeling in the hypertensive heart. Hypertension. 2010;55(2):270-6.

17. Brandt MC, Mahfoud F, Reda S, Schirmer SH, Erdmann E, Böhm $M$, et al. Renal sympathetic denervation reduces left ventricular hypertrophy and improves cardiac function in patients with resistant hypertension. J Am Coll Cardiol. 2012;59(10):901-9.

18. Levy D, Larson MG, Vasan RS, Kannel WB, Ho KK. The progression from hypertension to congestive heart failure. JAMA. 1996;275(20):1557-62.

19. van Dalen BM, Kauer F, Vletter WB, Soliman OI, van der Zwaan $\mathrm{HB}$, Ten Cate FJ, et al. Influence of cardiac shape on left ventricular twist. J Appl Physiol (1985). 2010;108(1):146-51.

20. Takeuchi M, Otsuji Y, Lang RM. Evaluation of left ventricular function using left ventricular twist and torsion parameters. Curr Cardiol Rep. 2009;11(3):225-30.

21. Cameli M, Lisi M, Righini FM, Massoni A, Mondillo S. Left ventricular remodeling and torsion dynamics in hypertensive patients. Int J Cardiovasc Imaging. 2013;29(1):79-86.

22. van Dalen BM, Tzikas A, Soliman OI, Kauer F, Heuvelman HJ, Vletter WB, et al. Left ventricular twist and untwist in aortic stenosis. Int J Cardiol. 2011;148(3):319-24.

23. Dong SJ, Hees PS, Siu CO, Weiss JL, Shapiro EP. MRI assessment of LV relaxation by untwisting rate: a new isovolumic phase measure of tau. Am J Physiol Heart Circ Physiol. 2001;281(5):H2002-9.

24. Bhatt DL, Kandzari DE, O’Neill WW, D'Agostino R, Flack JM, Katzen BT, et al. A controlled trial of renal denervation for resistant hypertension. N Engl J Med. 2014;370(15):1393-401.

25. Azizi M, Sapoval M, Gosse P, Monge M, Bobrie G, Delsart P, et al. Optimum and stepped care standardised antihypertensive treatment with or without renal denervation for resistant hypertension (DENERHTN): a multicentre, open-label, randomised controlled trial. Lancet. 2015;385(9981):1957-65. 